Optical and UV Spectroscopy of the Peculiar RS CVn System, RT Lacertae.

David P. Huenemoerder ${ }^{l}$

Department of Astronomy

Pennsylvania State University

University Park, PA 16802

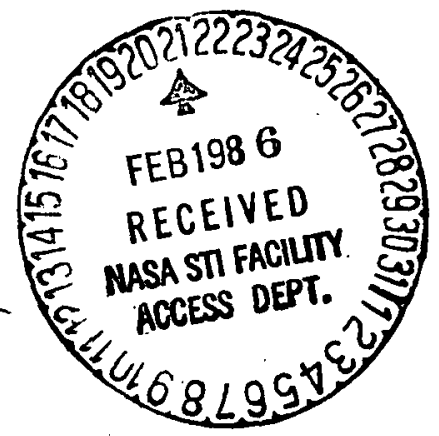

and

Samuel C. Barden

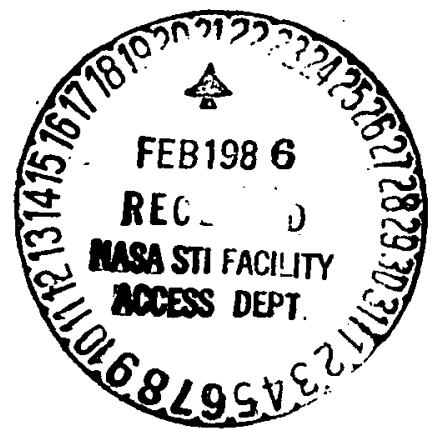

Kitt Peak National Observatory

National Optical Astronomy Observätories ${ }^{2}$

950 N. Cherry Ave.

P.O. Box 26732

Tucson, AZ 85726

Received

9 OCTOBER 1985

$I_{\text {Guest }}$ Observer with the International Ultraviolet Explorer satellite, which is sponsored and operated by the National Aeronautics and space Administration, the Science Research Council of the United Kingdom, and the European Space Agency.

${ }^{2}$ operated by the Association of Universities for Research in Astronomy, Inc., under contract with the National science Foundation. 
Optical and UV Spectroscopy of the Peculiar RS CVn System, RT Lacertae.

David P. Huenemoerder ${ }^{1}$

Department of Astronomy, Pennsylvania State University

and

Samuel C. Barden ${ }^{2}$

Kitt Peak National observatory

National optical Astronomy observatories

\section{ABSTRACT .}

We have obtained spectra in the $\mathrm{H}$-alpha and $\mathrm{H}$-beta regions of the peculiar double-lined RS CVn binary, RT. Lacertae, in the fall of 1984. Limited IUE long wavelength low and high resolution spectra were obtained concurrently. The ground based spectra have shown an asymmetry with orbital phase in the H-alpha profile. H-beta profiles were consistent with the same effect. One hemisphere showed excess emission and the other excess absorption, with a broad Gaussian emission component superposed upon the excess $\mathrm{H}$-alpha line. We derived an improved radial velocity curve, giving a better determined mass ratio and geometry. This combined with the radii implied by the 
rotational broadening of the spectra, showed one component to be $80-90 \%$ filling the equilibrium Roche surface. The two-faced nature is therefore very likely due to mass transfer from the contact component impacting upon its companion.

Low resolution ultraviolet data. showed that the supposed cooler component is bluer than its companion. This is also evidence of a scattering shell or cloud produced by the splash of a gas stream. High resolution ultraviolet data taken during secondary eclipse showed Mg II emission strength which decreased more slowly than the area visible, implying either a contribution from the contact component as well, or spatial inhomogeneities on the surface of the occulted star. The phase behavior of the low resolution data support the former situation, indicating "traditional" chromospheric activity as well.

1 Guest observer with the International Ultraviolet Explorer satellite, which is sponsored and operated by the National Aeronautics and Space Administration, the Science Research Council of the United Kingdom, and the European Space Agency.

${ }^{2}$ Operated by the Association of Universities for Research in Astronomy, Inc., under contract with the National Science Foundation. 
I. Introduction.

RT Lacertae has been classified as an RS CVn system by Hall (1976). Its fundamental characteristics and history in the literature were presented by Huenemoerder (1985) and the references therein. This paper is an extension of that work, in which we expand the data base of high quality CCD spectra of the $\mathrm{H}$-alpha and $\mathrm{H}$-beta regions. In addition, we have obtained spectra in the range $190 \mathrm{~nm}$ to $320 \mathrm{~nm}$ using the International Ultraviolet Explorer (IUE) in both the low and high dispersion modes. The IUE data were acquired concurrently with the ground based data.

We have used the same analysis technique as in Huenemoerder (1985) and Barden (1985), which derives radial velocities, rotational velocities, and relative intensity weights by fitting standard star spectra to the binary spectrum. From the velocities determined, we derive an improved radial velocity curve giving a more accurate orbital solution and mass ratio. The previous curve was due to Joy (1931) and rated a "d" in Batten's (1978) catalog. Other values for the masses, from an unpublished radial velocity curve determined by Popper, are given by popper and Ulrich (1977), but without any error estimates. From our spectra we also determined the stellar radii from the rotational velocity, known period, and the assumption of synchronous rotation. The resulting geometry and mass ratio has the hotter component filling $80 \%-90 \%$ of the equilibrium Roche radius. Another result of the fitting procedure is an assessment of the excess emission or absorption in the Balmer lines, compared to the standard stars. Most coverage was at H-alpha, which showed a two-faced behavior with phase; one hemisphere displayed excess emission and the other excess absorption. H-beta spectra were more 
sparse in phase coverage, but were consistent with the same trend. These observations suggested that the hotter component was indeed losing matter from the inner Lagrangian point.

LOW resolution IUE exposures were made during secondary eclipse and near quadrature. The low resolution data showed a bluer continuum when the cooler and fainter star was visible. This is in agreement with the photometry of Milone (1977). In the high resolution data, only the Mg II lines were visible.

II. Results.

All optical observations were made at Kitt Peak National Observatory (KPNO) with the coude feed telescope and spectrograph system, using camera five, grating $B$, and the Texas Instruments $C C D$. Observations were made primarily at $\mathrm{H}$-alpha, with a few exposures at H-beta also. The spectral resolutions were $0.05 \mathrm{~nm}$ at $\mathrm{H}$-alpha and $0.03 \mathrm{~nm}$ at $\mathrm{H}$-beta, representing about two channels full-width-half-maximum of a Th-Ar comparison line at both wavelength ranges. The KPNO data presented in Huenemoerder (1985) have been included here to provide additional phase coverage in deriving the radial velocity curve and to monitor the evolution of any spectral changes. An observing log is given in Table 1 , which also includes the orbital phase using the ephemeris of Tunca, et al. (1983) (MinI $=\mathrm{JD}(\mathrm{Hel}) 2444873.3668+5.074001 \mathrm{E}$ ) , the measured radial velocities and the observed-minus-computed (o-c) values.

The absolute error in radial velocity, as determined from radial velocity standards, was $3.8 \mathrm{~km} \mathrm{~s}^{-1}$. A clear and complete description of our reduction procedure, which fits standard star spectra to the binary to derive radial velocities, rotational velocities, and intensity weights, can be found in Barden (1985). In short, all lines in the spectrum, 
except for regions explicitly excluded, are fit simultaneously. This avoids. much of the usual problems of blending, distortion, and noise in individual photospheric profiles. We will present the results here and refer the interested reader to the former reference for the details. (The standards used were HR7602, G8 IV, and HR7957, K0 IV.)

a. The radial velocity curve and orbital solution.

The radial velocity curve is shown in Figure 1. Each component was fit independently via least-squares techniques, assuming zero eccentricity and using the ephemeris of Tunca, et al. (1983). The solid curves plotted are the results of the fit, using the weighted mean systemic velocity from each component's solution. The various parameters and their standard errors are given in Table 2, as are the results of the orbital solution (also assuming zero eccentricity). Note that the radial velocity errors determined from the sharp lined single radial velocity standards $\left(3.8 \mathrm{~km} \mathrm{~s}^{-1}\right)$ are virtually identical to the deviation about the fit radial velocity curve $\left(3.9 \mathrm{~km} \mathrm{~s} \mathrm{~s}^{-1}\right)$. This shows that the composite, rotationally broadened spectra are determined with the same accuracy as single stars. We have adopted an inclination of 89 degrees as determined from the visual light curve by Eaton and Hall (1979). The radii were derived from the rotational velocities found in the spectral fits, the known period, and the assumption of synchronous rotation.

The mass ratio of RT Lac, according to Kopal (1978), yields an equilibrium Roche surface for the secondary of five solar radii as measured perpendicularly to the line joing the two stars and in the obrital plane: Thus, for the derived radius of the secondary, it is within $80 \%$ to $90 \%$ of filling equilibrium Roche surface. Primary 
refers to the more massive component, which is the cooler, smaller, and fainter star, in the RT Lac system. This means the secondary G9 star is occulted during primary eclipse. Remember that the radii are somewhat uncertain, since they were derived from rotational velocities found by fitting artificially broadened standard star spectra to the observed binary. The broadening function assumed spherical stars with a standard limb darkening coefficient. The components of a real semi- or nearly detached system will certainly deviate from sphericity and have a generally unknown limb darkening function. Our other spectroscopic evidence indicates that the RT Lac secondary is actually filling and overflowing its Roche surface.

\section{b. The Optical spectra.}

The H-alpha spectra and the excess profiles derived by subtracting the synthetic composite from the fits are shown in Figure 2. The four scans from Huenemoerder (1985) have been reproduced here also. The spectra are arranged in order of increasing orbital phase and have been corrected for systemic velocity and the earth's motion. The H-alpha line centers of the components are indicated. Sharp features are telluric water vapor lines. An excess absorption in H-alpha, which occurs redward of the K star's $\mathrm{H} \alpha$ line component, increases in strength from phase zero up to secondary eclipse (phase 0.5). As viewed from the other direction, at phases 0.6 through 0.8 , excess emission occurred from both components. A broad emission component is superposed upon all H-alpha spectra, as is easily seen at phases 0.452 and 0.655 . 
The H-beta spectra are shown in the same fashion in Figure 3 . Excess $H$-beta spectra generally resembled the $H$-alpha ones, except that the $G$ star showed little or no excess emission. There were no obvious long term changes in either the H-alpha or H-beta lines from 1983 to 1984.

The system geometry is shown from a polar view in Figure 4. The dotted contour represents the Roche surface. The direction of view for the observed phases are marked around the outer circle.

c. The IUE Satellite Data.

Several exposures were made with the IUE satellite observatory (Boggess, et al. $1978 \mathrm{a}, \mathrm{b}$ ) contemporaneously with the optical observations. Two consecutive long wavelength high resolution exposures were made as RT Lac went into secondary eclipse. The only features visible were the Mg II multiplet at $280 \mathrm{~nm}$. Low resolution long wavelength exposures were made during secondary eclipse and near quadratures. The Mg II line was saturated in most of these in order to expose the continuum. An observing log is given in Table 3 .

Limited photometry was also available from the IUE satellite, in the form of the fine error sensor (FES) counts and the conversion to visual magnitudes (Holm and Crabb, 1979). which are good to about 0.1 magnitude. An estimate of the $B-V$ color index is needed for the measure, and was taken from the light curve for 1983-1984 from Fried (1984), discussed in Henemoerder (1985). The results and data necessary for conversion are Iisted in Table. 4. 
III. Discussion.

Our improved orbital solution, coupled with the spectroscopically determined radii, has placed RT Lac on the verge of being only semi-detached, with the hotter, lower mass component nearly filling its Roche lobe. The spectroscopic evidence presented here has led us to the conclusion that it indeed is semi-detached, as previously postulated by Milone (1977), Eaton and Hall (1979), and Milone and Naftilan (1980). Their conclusion was based on photometric evidence, such as period changes, ellipticity and eclipse duration variations, an infrared excess, and nearness of the radius of the hotter star to the Roche limit. Some of these features could possibly be explained by a spot model, but they could not fit the light curves to their satisfaction.

Assuming that Roche lobe overflow is occurring, we can apply the dynamical theory of Lubow and shu (1975) to constrain the stream characteristics. For the mass ratio and geometry of RT Lac, the stream leaving the inner Lagrangian point has a small radius compared to the stellar dimensions, and impacts on the $k$ component with a velocity of about $500 \mathrm{~km} \cdot \mathrm{s}^{-1}$, The impact area would be viewed face on somewhere near phase 0.4 .

It is near this phase when the excess $\mathrm{H}$-alpha absorption becomes strong, with broad emission wings. The absorption is slightly 
redshifted from the velocity of the $\mathrm{K}$ star. One interpretation could be that we are viewing a hot, turbulent region through an intervening receding gas; that is, we are looking along the stream as it falls onto the $\mathrm{K}$ star. However, the observations of Huenemoerder (1985), indicate that the excess absorption remains right through secondary eclipse, even when the $K$ star is occulted and suggested the possibility of mass loss through the outer Lagrangian point.

The gas stream scenario is supported by analogy to known mass-transfer systems. Peters and Polidan (1984) have reported asymmetry with phase in the behavior of ultraviolet absorption lines in some Algol-type systems. Absorptions by high ionization states were seen at phases consistent with the visibility of a stream impact as predicted by the theory of Lubow and Shu (1975). Peters and Polidan (1984) concluded that at least one hemisphere of the mass gaining star was affected by the process, producing a high temperature accretion region. The stars in their study each had a hot companion providing a bright continuum against which to see the absorption lines. Such is not the case with RT Lac. Both components are cool stars, and no continuum was visible in four hour high dispersion long wavelength IUE exposures in an attempt to detect a similar effect here. An alternate test for high temperature regions in RT Lac would be a search for high excitation emission lines in low resolution short wavelength IUE exposures at the phases where $\mathrm{H}$-alpha and $\mathrm{H}$-beta showed excess absorption and emission.

The ultraviolet observations have confirmed a color inconsistency first noticed independently by Milone and Hall as reported by Milone (1968). It was discussed by Milone $(1976,1977)$ and by Eaton and Hall (1979); the component of lower surface brightness, and presumably temperature, was 
bluer. Low resolution long wavelength IUE observations also showed this. Subtraction of the observed absolute flux during secondary eclipse (G star visible) from that at quadrature provided an approximate spectrum of the $\mathrm{K}$ component. It was not only slightly. steeper in slope than the $G$ component, but brighter. At $300 \mathrm{~nm}$, the $\mathrm{K}$ star contributes two thirds of the total light, compared to only about one third near H-alpha. A continuum index was formed from the flux at $310 \mathrm{~nm}$ and compared to the visual magnitude as estimated from the fine error sensor (FES) on IUE and the (B-V) color index from the photometry of Fried (1984) and Evren, et al. (1985). Our FES data agreed with the 1982-83 light curves. From IUE observations made during secondary eclipse (G star visible) and at quadrature, the $K$ star was found to be nearly a magnitude bluer in a $(310-V)$ color index. In a $(310-V)$ versus (B-V) sequence made from the orbiting Astronomical Observatory catalog of code and Meade (1979), the spectral types formed a fairly well behaved sequence. The G9 component of RT Lac fell among the G9 to ko region, while the $\mathrm{Kl}$ star was among the $\mathrm{G} 8$ stars. It was clearly bluer both in $(B-V)$ and in the ultraviolet index. This blueness could be a signature of a scattering shell, such as that produced by accretion of a gas stream. This possibility was also suggested by Milone (1977).

Additional theoretical support for mass transfer is given by the work of Gilitiand (1985) and the references therein. His and other theoretical calculations and observations showed that periods of steady mass loss occur for all semi-detached systems when the mass losing star is less than about one solar mass. The contact component of RT Lac is about 0.8 solar masses. 
It is interesting to consider the relationship of RT LaC, SZ PSC and, current theory. SZ PSC has shown peculiar H-alpha behavior which has been attributed to visibility of active regions (Weiler, 1978; Ramsey and Nations, 1981) and to transient mass transfer (Bopp, 1981;

Huenemoerder and Ramsey, 1984). One component is near to $90 \%$ filling the Roche surface (Jakate, et al., 1976; Eaton et al., 1982). The other star in the system has a size such that the stream would impact upon it, according to the theory of Lubow and Shu (1975). While no excess H-alpha absorption was seen by Huenemoerder and Ramsey, there was a correlation between the profile shape and the phase, reminiscent to that in RT Lac. The masses of the components of SZ PSC are both just above a solar mass (Jakate, et al., 1976). This is in the region where mass transfer is unstable, according to theory (Wood, 1977; Gilliland, 1985). Thus, RT Lac and SZ PSC may be two key experiments in mass transfer in binary stars, representing the boundary between steady and transient flow. They may provide constraints needed to improve the theory. Though they are both difficult systems to obtain adequate phase coverage having periods very close to integrai days, they warrant attention, perhaps enough to encourage coordinated observations in the interest of obtaining good phase and wavelength coverage.

Whether some of the spectroscopic activity of RT Lac is due to the active chromosphere phenomenon of "typical" RS CVn stars is difficult to assess. The $\mathrm{K}$ star always seems to have excess $\mathrm{H}$-beta emission, while at H-alpha, it usually does. The $G$ star has excess H-alpha emission near phase 0.7 . This could be due to the same type of chromospheric activity as is seen in other RS CVn stars. Both 
components also seem to have some steady $\mathrm{Mg}$ II $\mathrm{h}$ and $\mathrm{k}$ emission. As the system went into secondary eclipse ( $K$ star occulted), the strength of the Mg II lines decreased, but not as quickly as the visible area of the $K$ star. Either the $G$ star also has some Mg II activity, or it is spatially inhomogeneous on the $\mathrm{K}$ star. The low resolution IUE data also show weaker but prominent emission during secondary eclipse, suggesting that both stars are active.

Another possible explanation for the strong variations in the excess $\mathrm{H}$-alpha profile is suggested by the calculations of Cram and Mullan (1985). Their models have shown that the presence of a chromosphere increases the strength of $\mathrm{H}$-alpha absorption in late type stars, unless the pressure is high enough to drive the lines into emission. The extreme variations in H-alpha could then represent large inhomogeneities in the temperature and density structure of the outer atmosphere of RT Lac. More extensive observations and modeling is needed to assess this possibility. In particular, simultaneous H-alpha and H-beta spectra with good phase coverage are needed, coupled with chromospheric modeling similar to Cram and Mullan's for both lines, but tuned to the parameters of RT Lac.

The consistency of the data with both theory and other observations strongly suggest that RT Lac is a mass transfer system, and that this is the primary cause of its activity. Though the system is complicated, it may provide a useful example for studies of evolution of low mass binary stars. 
Acknowledgment

We would like to thank Dr. Lawrence Ramsey for critical comments on the presentation of these results. We also wish to thank the staff at kitt Peak and IUE for their assistance during the observing runs.

Funding for this research was provided by NASA grant NAG 5-405 and. NSF grant AST83-17867 to The Pennsylvania State University. 
Table 1

KPNO Observing Log

\begin{tabular}{|c|c|c|c|c|c|c|c|c|c|}
\hline UT & Date & $\&$ & time & phase & region & $v(K)^{*}$ & $O-C$ & $v(G)$ & $O-C$ \\
\hline 20 & sep & 84 & $\begin{array}{l}2: 31 \\
4: 35 \\
8: 10\end{array}$ & $\begin{array}{l}0.868 \\
0.885 \\
0.914\end{array}$ & $\begin{array}{l}\text { ta } \\
\text { pha } \\
\text { pha }\end{array}$ & $\begin{array}{l}-8 \\
-8 \\
-7\end{array}$ & $\begin{array}{l}6.5 \\
5.2 \\
4.6\end{array}$ & $\begin{array}{r}25.4 \\
17.8 \\
0.5\end{array}$ & $\begin{array}{l}-4.7 \\
-3.7 \\
-4.3\end{array}$ \\
\hline 21 & sep & 84 & $\begin{array}{l}2: 50 \\
6: 37\end{array}$ & $\begin{array}{l}0.067 \\
0.098\end{array}$ & $\begin{array}{l}\text { H-alpha } \\
\text { H-beta }\end{array}$ & $\begin{array}{l}-34.4 \\
-16.8\end{array}$ & $\begin{array}{r}-2.4 \\
6.2\end{array}$ & $\begin{array}{l}-109.2 \\
-121.4\end{array}$ & $\begin{array}{l}-9.2 \\
-2.2\end{array}$ \\
\hline 8 & oct & 84 & $\begin{array}{l}3: 17 \\
5: 30 \\
7: 18\end{array}$ & $\begin{array}{l}0.421 \\
0.440 \\
0.454\end{array}$ & $\begin{array}{l}\mathrm{H}- \\
\mathrm{H}- \\
\mathrm{H}-\end{array}$ & $\begin{array}{l}-29.6 \\
-22.4 \\
-34.6\end{array}$ & $\begin{array}{r}-1.2 \\
11.7 \\
3.9\end{array}$ & $\begin{array}{r}-101.8 \\
-87.8 \\
-86.6\end{array}$ & $\begin{array}{r}5.8 \\
7.6 \\
-0.7\end{array}$ \\
\hline 9 & oct. & 84 & $\begin{array}{l}2: 32 \\
4: 35 \\
7: 47\end{array}$ & $\begin{array}{l}0.612 \\
0.629 \\
0.655\end{array}$ & $\begin{array}{l}\mathrm{H}- \\
\mathrm{H}- \\
\mathrm{H}-\end{array}$ & $\begin{array}{l}-89.8 \\
-89.0 \\
-99.6\end{array}$ & $\begin{array}{r}-1.9 \\
3.0 \\
-2.2\end{array}$ & $\begin{array}{l}22.8 \\
35.7 \\
39.1\end{array}$ & $\begin{array}{r}3.0 \\
7.0 \\
-1.2\end{array}$ \\
\hline 10 & oct & 84 & $\begin{array}{l}2: 11 \\
5: 18\end{array}$ & $\begin{array}{l}0.8 \\
0.8\end{array}$ & $\begin{array}{l}\mathrm{H}- \\
\mathrm{H}-\end{array}$ & $\begin{array}{l}-99.3 \\
-99.4\end{array}$ & $\begin{array}{l}4.0 \\
0.5\end{array}$ & .1 & $\begin{array}{l}-5.8 \\
-6.9\end{array}$ \\
\hline 15 & Oct & 83 & $\begin{array}{l}9: 32 \\
9: 44\end{array}$ & $\begin{array}{l}0.720 \\
0.722\end{array}$ & $\begin{array}{l}\text { ha } \\
\text { ha }\end{array}$ & $\begin{array}{l}-103.0 \\
-103.9\end{array}$ & 2.7 & $\begin{array}{l}51.3 \\
51.8\end{array}$ & $\begin{array}{l}-6.6 \\
-6.3\end{array}$ \\
\hline 16 & oct & 83 & $\begin{array}{l}3: 21 \\
3: 56 \\
6: 30\end{array}$ & $\begin{array}{l}0.866 \\
0.871 \\
0.892\end{array}$ & $\begin{array}{l}\text { ta } \\
\text { ta } \\
\text { pha }\end{array}$ & $\begin{array}{l}-90.5 \\
-89.1 \\
-81.7\end{array}$ & $\begin{array}{l}2.6 \\
2.9 \\
5.2\end{array}$ & $\begin{array}{l}28.2 \\
25.6 \\
14.3\end{array}$ & $\begin{array}{l}-2.8 \\
-3.1 \\
-3.3\end{array}$ \\
\hline & oct & 83 & $5: 12$ & 0.078 & $\mathrm{H}-\mathrm{a}$ & -28.4 & 0.3 & -115.4 & 8.4 \\
\hline 1 & oct & 83 & $\begin{array}{l}2: 44 \\
3: 17\end{array}$ & $\begin{array}{l}0.452 \\
0.457\end{array}$ & $\begin{array}{l}\mathrm{H}-\mathrm{al} \text { l } \\
\mathrm{H}-\mathrm{al}\end{array}$ & $\begin{array}{l}-45.0 \\
-32.8\end{array}$ & $\begin{array}{r}-7.2 \\
6.6\end{array}$ & $\begin{array}{l}-95.1 \\
-87.2\end{array}$ & $\begin{array}{l}-7.8 \\
-3.3\end{array}$ \\
\hline
\end{tabular}

*Absolute error $=3.8 \mathrm{~km} \mathrm{~s}^{-1}$ 
Table 2

Radial Velocity and orbital solution

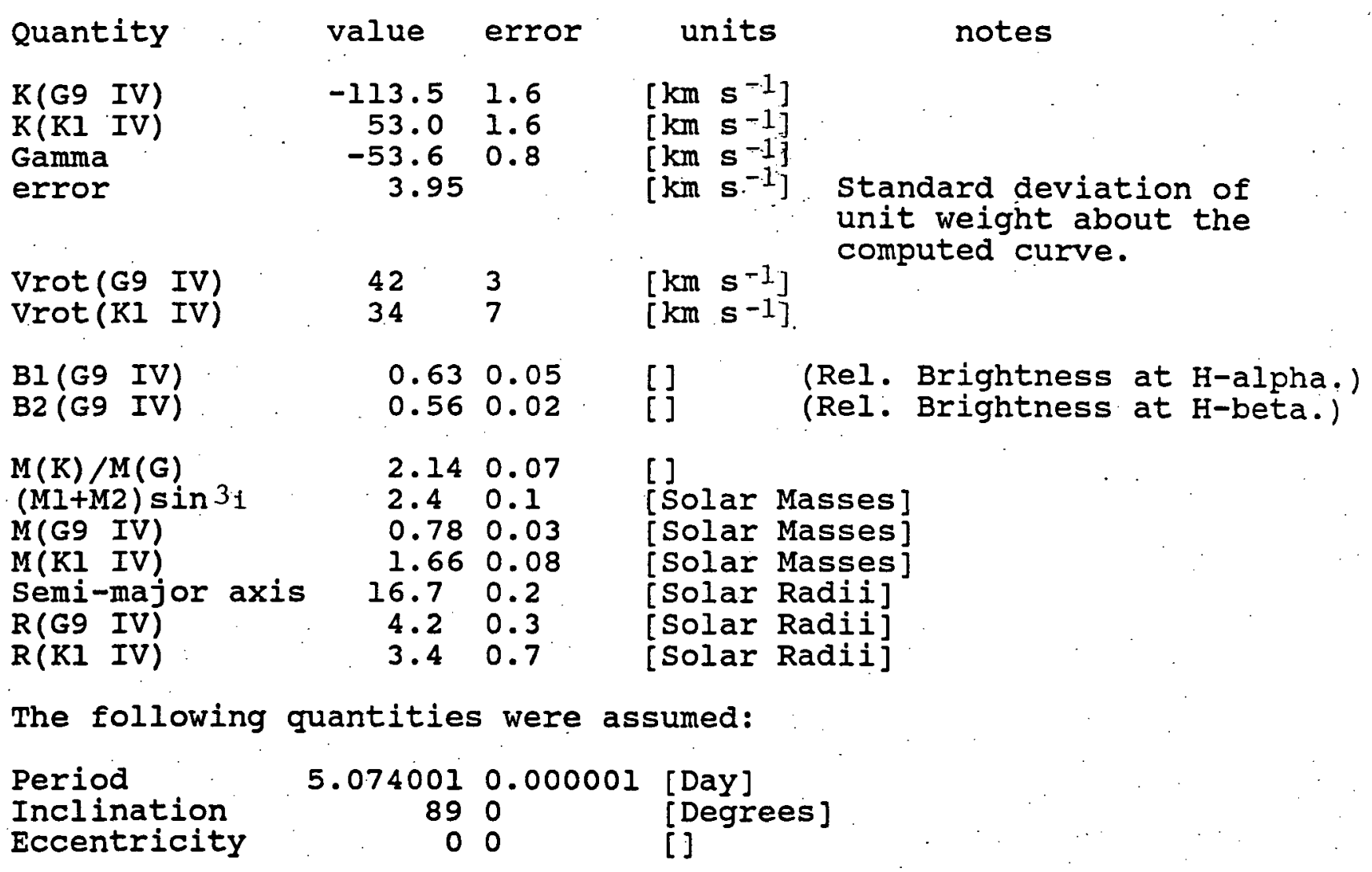


Table 3

\begin{tabular}{rlllllll}
\multicolumn{7}{c}{ IUE Observing Log } & \\
image & cam & disp & UT date \& time & texp & phase \\
4244 & LWP & Hi & 17 Sep 84 & $21: 39$ & 210 & 0.448 \\
4245 & LWP & Hi & 18 Sep 84 & $1: 41$ & 185 & 0.479 \\
4253 & LWP & LO & 19 Sep 84 & $10: 35$ & 35 & 0.739 \\
4254 & LWP & LO & 19 Sep 84 11:43 & 15 & 0.749 \\
24006 & SWP & LO & 19 Sep 84 12:04 & 60 & 0.755 \\
4255 & LWP & LO & 19 Sep 84 & $13: 10$ & 15 & 0.761 \\
4524 & LWP & LO & 8 Oct 84 & $9: 42$ & 20 & 0.475 \\
4525 & LWP & LO & 8 Oct $84.10: 39$ & 15 & 0.483 \\
4526 & LWP & LO & 8 Oct 84 & $11: 26$ & 15 & 0.489 \\
4527 & LWP & LO & 8 Oct $8412: 14$ & 15 & 0.496
\end{tabular}

The date, time and phase are given for mid-exposure. The exposure times are in minutes. 


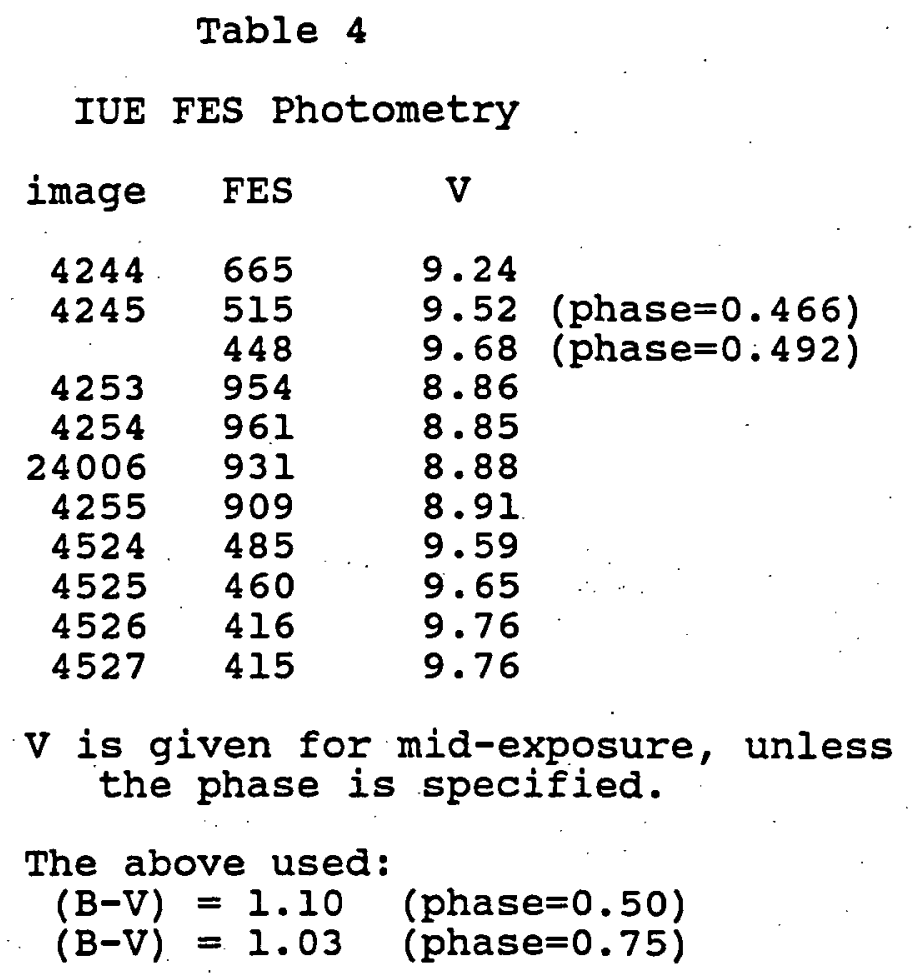




\section{References}

Barden, S. C. 1985, Ap. J., 295, 162.

Batten, A. H., Fletcher, J. M., and Mann, P. J. 1978, Pub. Dom. Astrophys. Obs., 15, No. 5 .

Boggess, A., et al. 1978a, Nature, 275, 372 .

Boggess, A., et al. 1978b, Nature, $275,377$.

Bopp, B. W. 1981, A. J., 86, 771 .

Code, A. D., and Meade, M. R. 1979, Ap. J. Suppl., 39, 195:

Cram, L. E., and Mullan, D. J. 1985, Ap. J., 294, 626.

Eaton, J. A., and Hall, D. S. 1979, Ap. J., 227, 907.

Eaton, J. A., Scaltriti, F., Cerruti-Sola, M., Sorma, M. B. K., Ausekar, B. D. , Catalano, S., and Rodono, M. 1982, Ap. Sp. Sc., $82,289$.

Evren, S., Tunca, Z., Ibanoglu, C., and Tumer, O. 1985, Ap. Sp. Sc., 108,383 .

Fried, R. E. 1984, Private communication.

Gilliland, R. L. 1985, Ap. J., 292, 522 .

Hall, D. S. 1976, in "Multiple Periodic Variable Stars", IAU Colloquium No. 29, edited by :. S. Fitch (Reidel, Dordrecht).

Holm, A., and Crabb, W. 1979, NASA IUE Newsletter, No. 7, 40.

Huenemoerder, D.' P. 1985; A. J., 90, 499.

Huenemoerder, D. P., and Ramsey, L. W. 1984, A. J., $89,549$.

Jakate, S., Bakos, G. A., Fernie, J. D., and Heard, J. F. 1976,

A. J., 81,250 .

Joy, A. H. 1931, Ap. J., 74, 101.

Kopal, Z. 1978, "Dynamics of close Binary systems", (Reidel, Dordrecht).

Lubow, S. H., and Shu, F. H. 1975, Ap. J., 198, 383.

Milone, E. F. 1968, A. J. 68, 708.

Milone, E. F. 1976, Ap. J. Suppl.,31, 93.

Milone, E. F. 1977, A. J., 82, 998. 
Milone, E. F. and Naftilan, S. A. 1980, in "Close Binary Stars: observations and Interpretations", IAU Symposium No. 88, edited by M. J. Plavec and R. K. Ulrich (Reidel, Dordrecht).

Peters, G. J.; and Polidan, R. S. 1984, Ap. J., 283, 745.

Popper, D. M. and Ulrich, R. K. 1977, Ap. J., 212, 431.

Ramsey, L. W. and Nations, H. L. 1981, P.A.S.P., 93, 732.

Tunca, Z., Ibanoglu, C.; Tumer, O., Ertan, A. Y., and Evren, S. 1983, Ap. Sp. Sc., 93, 431.

Weiler, E. J. 1978, M.N.R.A.S., 182, 77.

Wood, P. R. 1977, Ap. J., 217, 530. 
Figure Captions.

Figure 1.

The radial velocity curve for RT Lac is shown. The solid curves are the least squares fits to the velocities of the $G$ component $(*)$ and $\mathrm{K}$ component $(+)$. Error bars represent a standard deviation of unit weight about the curve. All data were weighted equally.

Figure 2.

a) The H-alpha spectra are shown in order of increasing phase from top to bottom. The solid line is RT Lac and the dotted are the fitted composite spectra.

b) These are the excess H-alpha profiles, obtained by subtracting the dotted profiles from the solid ones in panel a. The central vertical line represents the rest $\mathrm{H}-\mathrm{alpha}$ line center. The vertical dashes above each spectrum mark the velocities of each component at that phase (the longer bar represents the primary $\mathrm{K}$ star).

Vertical offsets in $a$ and $b$ are 0.5 intensity units, with the continuum normalized to one. 
Figure 3 .

Like Figure 2, but for the H-beta region.

Figure 4.

This is a schematic polar view of RT Lac in proper scale. The stars are represented as solid circles and the Roche surface as a dotted contour. A stream, and presumed hot spot and circumstellar matter are sketched in with hash marks. Tic marks around the outer circle denote the direction of view at observed phases. Phase 0.0 is at the right and phases increase in the clockwise direction. 
Figure 1

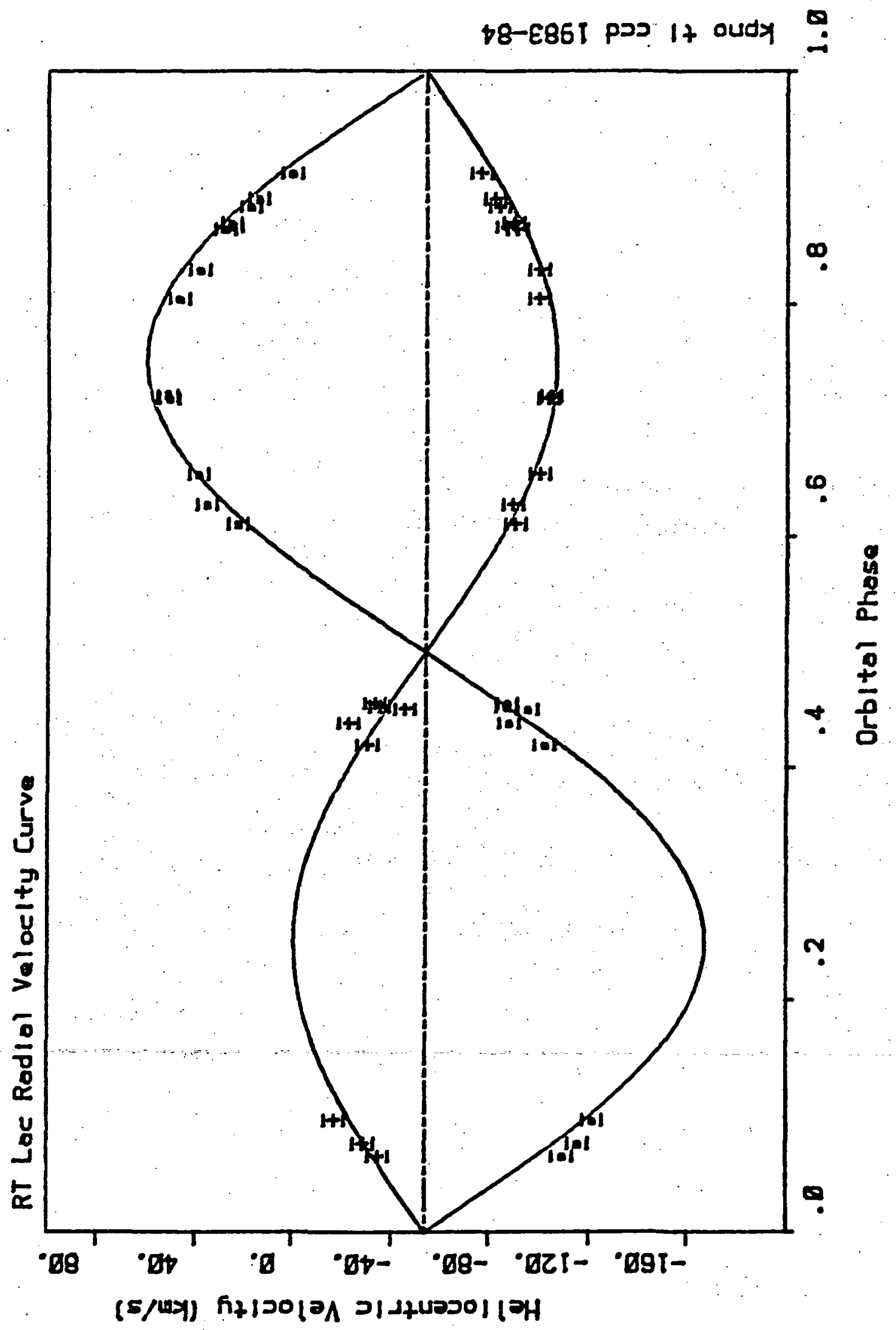


Figure 2a

phase

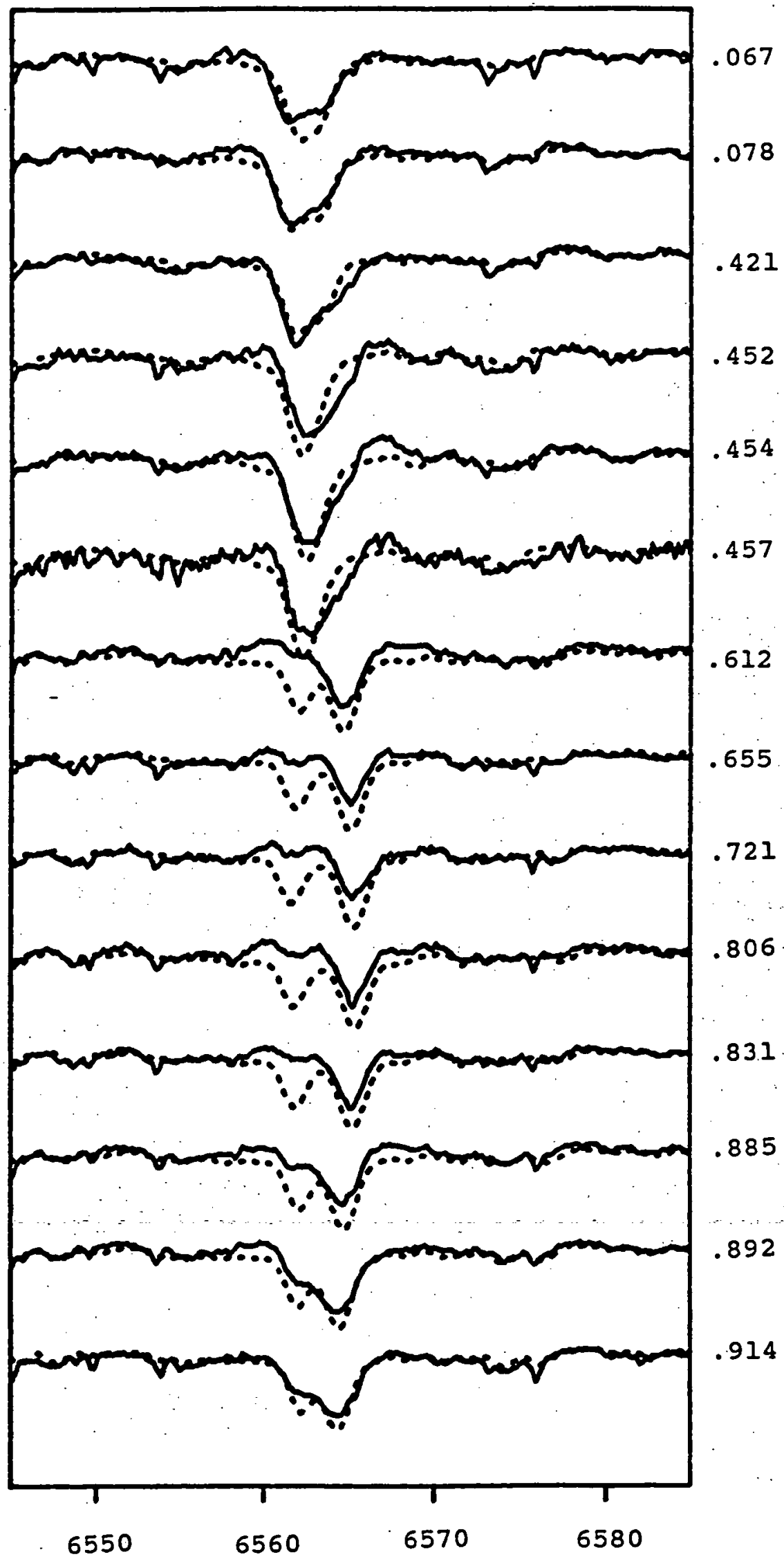

Wavelength 
Figure 2b

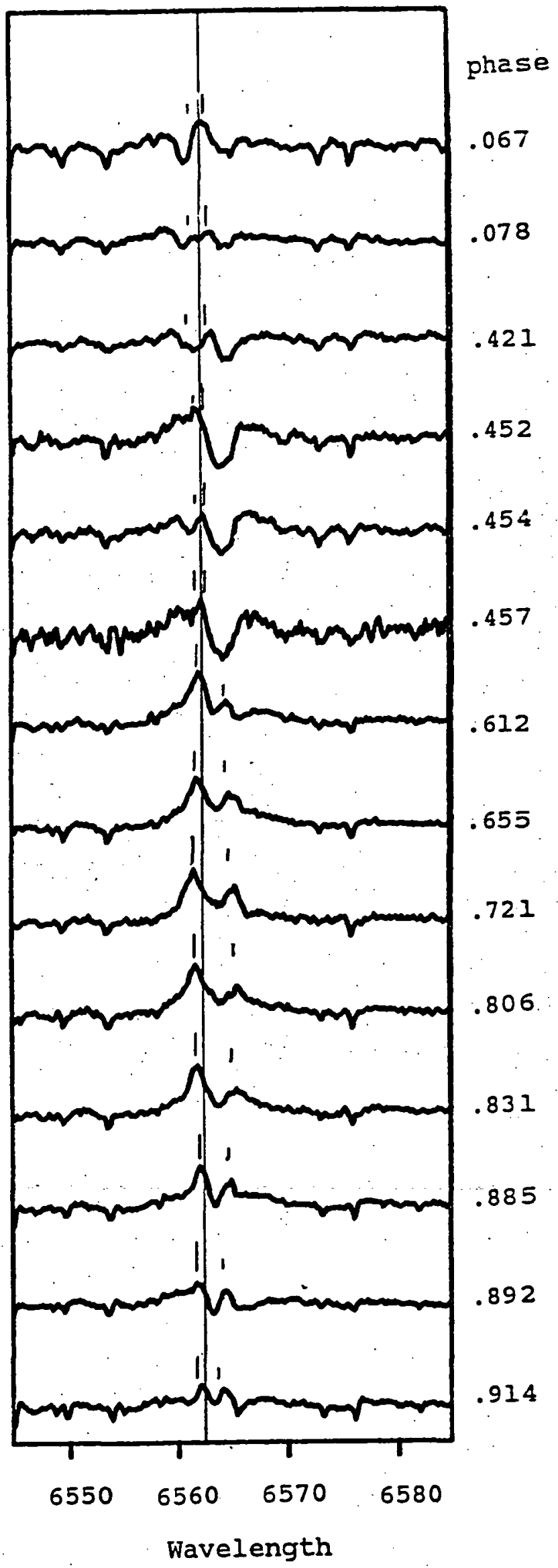


Figure $3 a$

phase

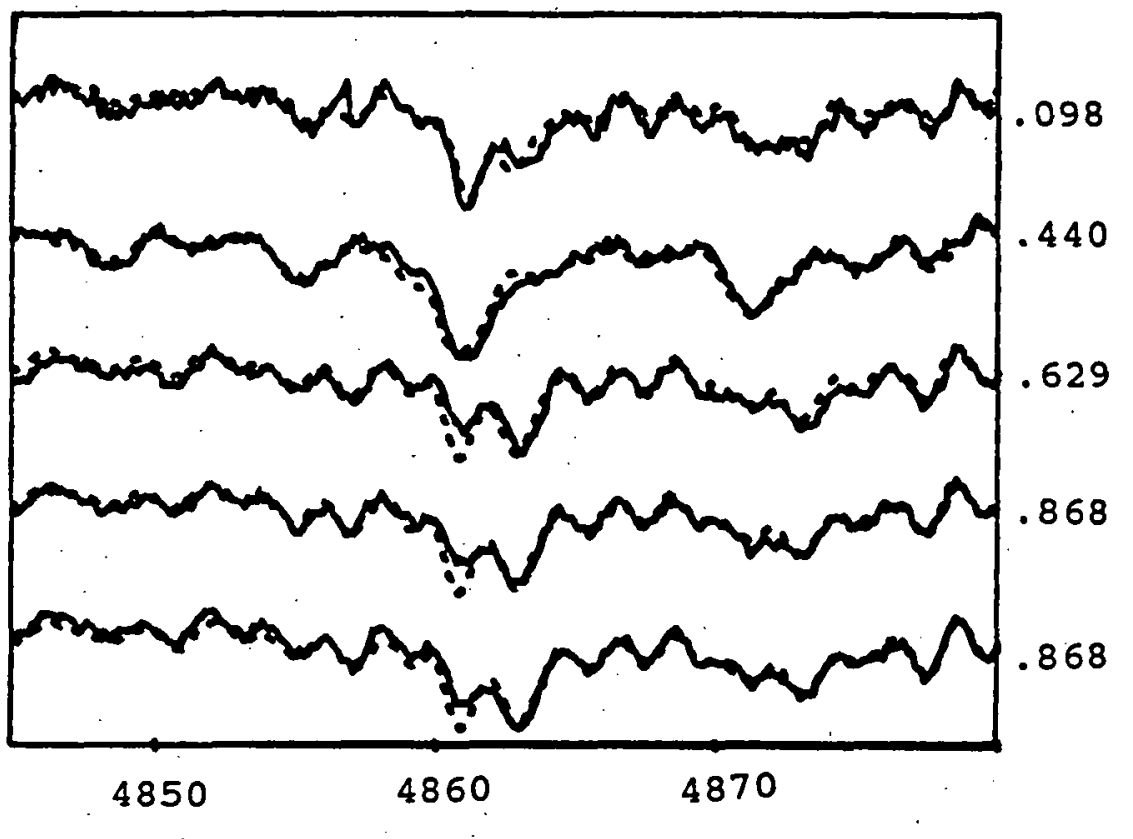

Wavelength.

Figure 30

phase

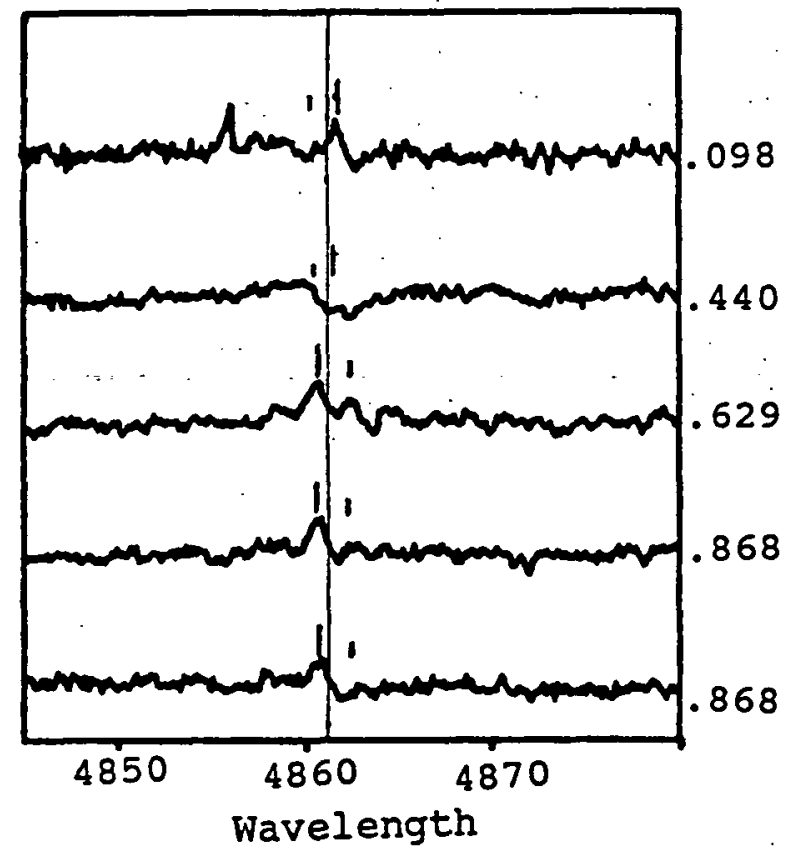


Figure 4

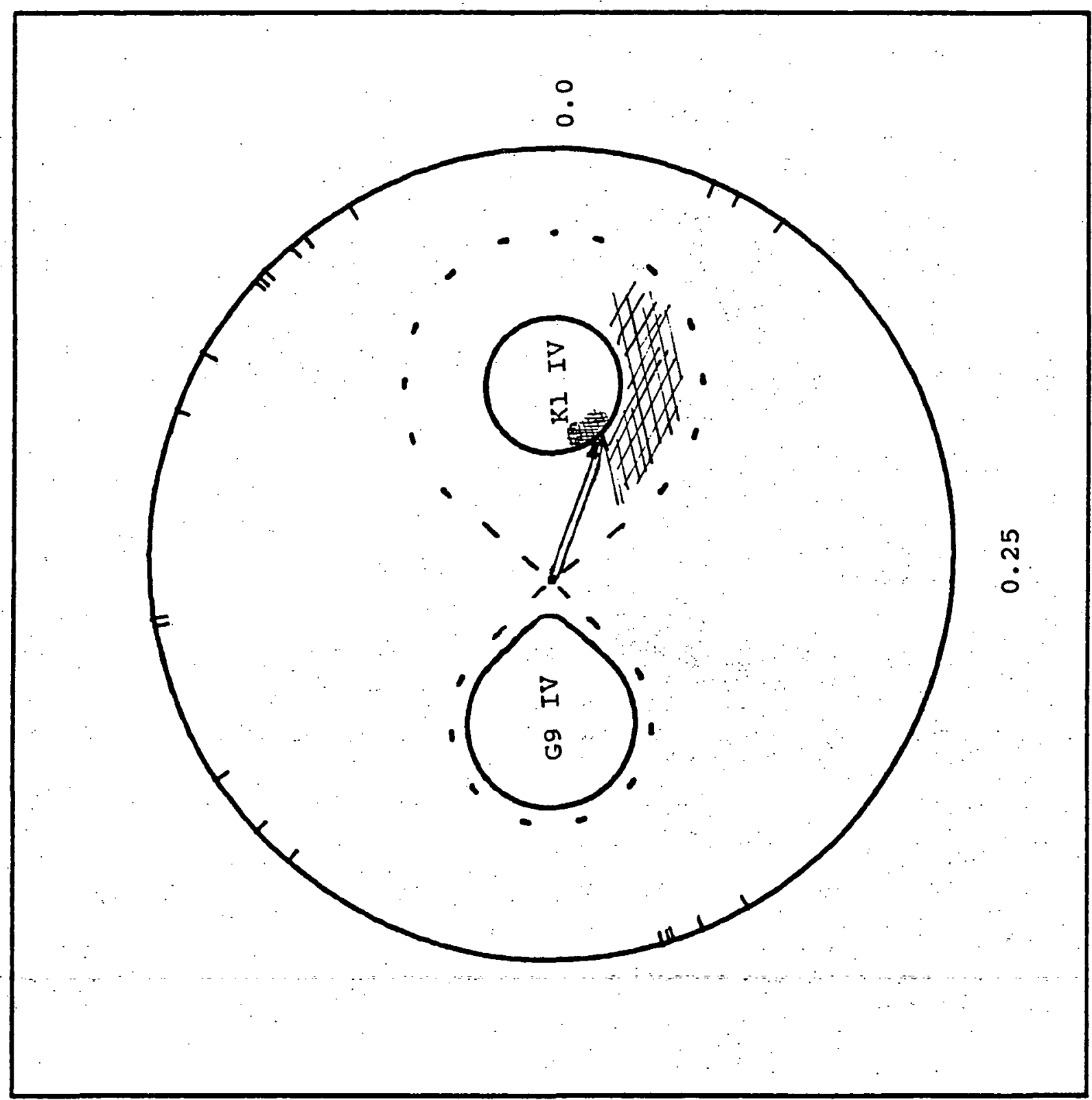


Authors' addresses:

\author{
Samuel C. Barden \\ Kitt Peak National Observatory \\ National optical Atronomy Observatories \\ 950 N. Cherry Ave. \\ P.O. BOX 26732 \\ Tucson, AZ 85726
}

David P. Huenemoerder

Department of Astronomy

Pennsylvania state University

University Park, PA 16802 